\title{
Things We Do for No Reason: Intermittent Pneumatic Compression for Medical Ward Patients?
}

\author{
Jürgen L Holleck, MD ${ }^{1,2 *}$; Craig G Gunderson, MD ${ }^{1,2}$
}

\begin{abstract}
'Department of Internal Medicine, Yale University School of Medicine, New Haven, Connecticut; ${ }^{2}$ Department of Medicine, Veterans Affairs
\end{abstract} Connecticut Healthcare System, West Haven, Connecticut.

The "Things We Do for No Reason" series reviews practices that have become common parts of hospital care but may provide little value to our patients. Practices reviewed in the TWDFNR series do not represent "black and white" conclusions or clinical practice standards, but are meant as a starting place for research and active discussions among hospitalists and patients. We invite you to be part of that discussion.

\section{CLINICAL SCENARIO}

A 74-year-old man with a history of diabetes and gastrointestinal bleeding two months prior, presents with nausea/vomiting and diarrhea after eating unrefrigerated leftovers. Body mass index is 25. Labs are unremarkable except for a blood urea nitrogen of $37 \mathrm{mg} / \mathrm{dL}$, serum creatinine of $1.6 \mathrm{mg} / \mathrm{dL}$ up from 1.3 , and white blood cell count of $12 \mathrm{~K} / \mu \mathrm{L}$. He is afebrile with blood pressure of $100 / 60 \mathrm{~mm} \mathrm{Hg}$. He lives alone and is fully ambulatory at baseline. The Emergency Department physician requests observation admission for "dehydration/gastroenteritis." The admitting hospitalist orders intermittent pneumatic compression (IPC) for venous thromboembolism (VTE) prophylaxis.

\section{BACKGROUND}

The American Public Health Association has called VTE prophylaxis a "public health crisis" due to the gap between existing evidence and implementation. ${ }^{1}$ The incidence of symptomatic deep venous thrombosis (DVT) and pulmonary embolism (PE) in hospitalized medical patients managed without prophylaxis is $0.96 \%$ and $1.2 \%$, respectively, ${ }^{2}$ whereas that of asymptomatic DVT in hospitalized patients is approximately $1.8 \% .^{2,3}$ IPC is widely used, and an international registry of 15,156 hospitalized acutely ill medical patients found that $22 \%$ of United States patients received IPC for VTE prophylaxis compared with $0.2 \%$ of patients in other countries. ${ }^{4}$

\footnotetext{
*Corresponding Author: Jürgen L. Holleck, MD; E-mail: Jurgen.Holleck@ va.gov; Telephone: 203-932-5711, ext. 4412.

Additional Supporting Information may be found in the online version of this article.
}

Received: June 1, 2018; Revised: October 11, 2018; Accepted: October 18, 2018

(C) 2019 Society of Hospital Medicine DOI 10.12788/jhm.3114

\section{WHY YOU MIGHT THINK IPC IS THE BEST OPTION FOR VTE PROPHYLAXIS IN MEDICAL WARD PATIENTS}

The main reason clinicians opt to use IPC for VTE prophylaxis is the wish to avoid the bleeding risk associated with heparin. The American College of Chest Physicians antithrombotic guideline 9th edition (ACCP-AT9) recommends mechanical prophylaxis for patients at increased risk for thrombosis who are either bleeding or at "high risk for major bleeding. " 5 The guideline considered patients to have an excessive bleeding risk if they had an active gastroduodenal ulcer, bleeding within the past three months, a platelet count below $50,000 / \mathrm{ml}$, or more than one of the following risk factors: age $\geq 85$, hepatic failure with INR $>1.5$, severe renal failure with GFR $<30 \mathrm{~mL}$ / $\mathrm{min} / \mathrm{m}^{2}$, ICU/CCU admission, central venous catheter, rheumatic disease, current cancer, or male gender. ${ }^{5}$ IPC also avoids the risk of heparin-induced thrombocytopenia, which is a rare but potentially devastating condition.

Prior studies have shown that IPC reduces VTE in high-risk groups such as orthopedic, surgical, trauma, and stroke patients. The largest systematic review on the topic found 70 studies of 16,164 high-risk patients and concluded that IPC reduced the rate of DVT from $16.7 \%$ to $7.3 \%$ and PE from $2.8 \%$ to $1.2 \%{ }^{6}$ Since the publication of this systematic review, an additional large randomized trial of immobile patients with acute stroke was published, which found a reduction in the composite endpoint of proximal DVT on screening compression ultrasound or symptomatic proximal DVT from $12.1 \%$ to $8.5 \%$. $^{7}$ Another systematic review of 12 studies of high-risk ICU patients found that IPC conferred a relative risk of $0.5195 \% \mathrm{Cl}$ : 0.20-1.23) for DVT, although this result was not statistically significant. ${ }^{8}$ Finally, a Cochrane review of studies that compared IPC combined with pharmacologic prophylaxis with pharmacologic prophylaxis alone in high-risk trauma and surgical patients found reduced PE for the combination. ${ }^{9}$

\section{WHY IPC MIGHT NOT BE AS HELPFUL IN MEDICAL WARD PATIENTS}

IPC devices are frequently not worn or turned on. A study at two university-affiliated level one trauma centers found IPC to be functioning properly in only $19 \%$ of trauma patients. ${ }^{10}$ In another study of gynecologic oncology patients, $52 \%$ of IPCs were functioning improperly and $25 \%$ of patients experienced some discomfort, inconvenience, or problems with external pneumatic compression. ${ }^{11}$ Redness, itching, or discomfort was 
TABLE 1. University of California (UC) San Diego "3 Bucket" Model Updated from the CHEST AT-8 Model

Low Risk: Observation status with expected LOS < 48 hours. Minor ambulatory surgery unless multiple strong risk factors, Medical patients ambulating in hall and not moderate or high risk. Ambulatory cancer patients admitted for short chemotherapy infusion.

No prophylaxis but ambulate and reassess intermittently

Moderate Risk: Most general, thoracic, open gynecologic or urologic surgery patients. Active cancer or past VTE or known thrombophilia in medical patients with LOS $>48$ hours. Medical patients with decrease in usual ambulation AND VTE risk factors: myocardial infarction, stroke, congestive heart failure, pneumonia, active inflammation/infection, dehydration, age $>65$.

High Risk: Hip or knee arthroplasty, hip fracture surgery, multiple major trauma, spinal cord injury or major spinal surgery, abdominal-pelvic IPC and pharmacologic prophylaxis (UFH or LMWH) surgery for cancer.

cited by $26 \%$ of patients, and patients removed IPCs $11 \%$ of the time when nurses left the room. ${ }^{11,12}$ In another study, skin breakdown occurred in 3\% of IPC patients as compared with $1 \%$ in the control group. ${ }^{7}$

Concerns about a possible link between IPC and increased fall risk was raised by a 2005 report of 40 falls by the Pennsylvania Patient Safety Reporting System, ${ }^{13}$ and IPC accounted for 16 of 3,562 hospital falls according to Boelig and colleagues. ${ }^{14}$ Ritsema et al. found that the most important perceived barriers to IPC compliance according to patient surveys were that the devices "prevented walking or getting up" (47\%), "were tethering or tangling" (25\%), and "woke the patient from sleep" $(15 \%){ }^{15}$

IPC devices are not created equally, differing in "anatomical location of the sleeve garment, number and location of air bladders, patterns for compression cycles and duration of inflation time and deflation time. ${ }^{16}$ Comparative effectiveness may differ. A study comparing a rapid inflation asymmetrical compression device by Venaflow with a sequential circumferential compression device by Kendall in a high-risk post knee replacement population produced DVT rates of $6.9 \%$ versus $15 \%$, respectively $(P=.007) .{ }^{16,17}$ Furthermore, the type of sleeve and device may affect comfort and compliance as some sleeves are considered "breathable."

Perhaps most importantly, data supporting IPC efficacy in general medical ward patients are virtually nonexistent. Ho's meta-analysis of IPC after excluding surgical patients found a relative risk (RR) of $0.53(95 \% \mathrm{Cl}: 0.35-0.81, P<.01)$ for DVT in nine trials and a nonstatistically significant RR of $0.64(95 \% \mathrm{Cl}$ : 0.29-1.42. $P=.27)$ for $P E$ in six trials. ${ }^{6}$ However, if high-risk populations such as trauma, critical care, and stroke are excluded, then the only remaining study is a letter to the editor published in 1982 that compared 20 patients with unstable angina treated with IPC with 23 controls and found a nonsignificant reduction in screened VTE. ${ }^{18}$ Given the near complete lack of data supporting IPC in medical patients, the ACCP-AT9 guideline rates the strength of evidence recommendation to use IPC only in medical patients who are currently bleeding or at high risk of major bleeding as "2C," which is defined as "weak recommendation" based on "low-quality or very low-quality evidence." 19 Similarly, the latest American College of Physicians guidelines (2011) recommend pharmacologic prophylaxis for medical patients rather than IPC, except when bleeding risk outweighs the likely benefit of pharmacologic prophylaxis. The guidelines specifically recommend against graduated com- pression stockings given the lack of efficacy and increased risk of skin breakdown. ${ }^{20}$

IPC is expensive. The cost for pneumatic compression boots is quoted in the literature at $\$ 120$ with a range of $\$ 80-\$ 250 .{ }^{21}$ Furthermore, patients averaged 2.5 pairs per hospitalization. ${ }^{22}$ An online search of retail prices revealed a pair of knee-length Covidien 5329 compression sleeves at $\$ 299.19$ per pair ${ }^{23}$ and knee-length Kendall 7325-2 compression sleeves at $\$ 433.76$ per pair ${ }^{24}$ with pumps costing $\$ 7,518.07$ for Venodyne $610 \mathrm{Ad}$ vantage ${ }^{25} \$ 6,965.98$ for VenaFlow Elite, ${ }^{26}$ and $\$ 5,750.50$ for Covidien 29525700 series Kendall SCD. ${ }^{27}$ However, using these prices would be overestimating costs given that hospitals do not pay retail prices. A prior surgical cost/benefit analysis used a prevalence of $6.9 \%$ and a $69 \%$ reduction of DVT. ${ }^{28}$ However, recent data showed that VTE incidence in 31,219 medical patients was only $0.57 \%$ and RR for a large VTE prevention initiative was a nonsignificant $10 \%$ reduction. ${ }^{29}$ Even if we use a VTE prevalence of $1 \%$ for the general medical floor and $0.5 \%$ RR reduction, 200 patients would need to be treated to prevent one symptomatic VTE and would cost about $\$ 24,000$ for IPC sleeves alone (estimating $\$ 120$ per patient) without factoring in additional costs of pump purchase or rental and six additional episodes of anticipated skin breakdown. In comparison, the cost for VTE treatment ranges from $\$ 7,712$ to $\$ 16,644.30$

\section{WHAT SHOULD WE DO INSTEAD?}

First, one should consider if VTE prophylaxis is needed based on risk assessment. According to the Agency for Healthcare Research and Quality (AHRQ), the most widely used risk stratification model is the University of California San Diego "3 bucket model" (Table 1) derived from tables in ACCP-AT8 guidelines. ${ }^{31}$ The Caprini risk assessment model has been validated for surgical patients, but AHRQ offers caveats related to the complexity of the tool, the difficulty many sites have integrating it into order sets, and the negative experience of the Michigan Hospital Medicine Safety Consortium. The consortium enrolled 43 hospitals with the great majority using the Caprini risk assessment model, but it failed to reduce VTE in medical patients. ${ }^{31}$ Alternatively, the ACCP-AT9 guidelines recommend the Padua prediction score for risk assessment of medical patients (Table 2). VTE occurs in $0.3 \%$ of low-risk patients (Padua score $<4$ ) and $11.0 \%$ of high-risk patients (Padua score $\geq 4$ ). If IPC is used in the low-risk populations with a predicted VTE rate of 0.3 , then 666 patients would need to be treated to prevent one VTE. Treating 666 patients would cost 
TABLE 2. Padua Prediction Score to Assess Risk Factors for VTE in Hospitalized Medical Patients (score $<4=$ low risk; score $\geq 4$ = high risk)

\begin{tabular}{lc}
\hline Risk Factor & Points \\
\hline Active cancer & 3 \\
\hline Previous VTE (excluding superficial vein thrombosis) & 3 \\
\hline Reduced mobility & 3 \\
\hline Thrombophilia & 3 \\
\hline Trauma and/or surgery in past month & 2 \\
\hline Age $\geq 70$ & 1 \\
\hline Heart or respiratory failure & 1 \\
\hline Acute myocardial infarction or stroke & 1 \\
\hline Acute infection or rheumatologic disorder & 1 \\
\hline Obesity BMI $\geq 30$ & 1 \\
\hline Ongoing hormonal treatment & 1 \\
\hline
\end{tabular}

$\$ 79,920$ for IPC sleeves alone plus $\$ 5,500-\$ 7,500$ per pump and result in 20 additional episodes of skin breakdown. Therefore, IPC should be reserved for high-risk populations with contraindications to pharmacologic prophylaxis.

\section{RECOMMENDATIONS}

- The VTE risk of general medicine ward patients should be assessed, preferably with the " 3 bucket" or Padua risk assessment models.

- For low-risk patients, no VTE prophylaxis is indicated. Ambulation ought to be encouraged for low-risk patients.

- If prophylaxis is indicated, then bleeding risk should be assessed to determine a contraindication to pharmacologic prophylaxis. If there is excessive bleeding risk, then treatment with IPC may be considered even though there are only data to support this in high-risk populations such as surgical, stroke, trauma, and critical care patients.

- If using IPC, then strategies that ensure compliance and consider patient comfort based on type and location of sleeves should be implemented.

- Combined IPC and pharmacologic prophylaxis should be used for high-risk trauma or surgical patients.

\section{CONCLUSIONS}

No current evidence supports IPC efficacy in general medical ward patients despite its widespread use; thus, prospective trials in this population are needed. Given costs, potential side effects, and uncertain efficacy in general medical ward patients, IPC should be reserved for surgical, trauma, critical care, or stroke patients. It may be considered for moderate to highrisk medical patients with excessive bleeding risk. Our clinical scenario patient bled within the past three months (odds ratio for bleeding 3.64; 95\% Cl, 2.21-5.99). ${ }^{32}$ On the basis of the in- creased risk, a dutiful hospitalist might be tempted to order IPC. However, given that our patient is ambulatory, is toileting frequently, and has an expected observation stay of less than 48 hours, he is considered low risk for VTE (Table 1). Additionally, his Padua score of two confirms his low risk status (Table 2). No VTE prophylaxis would be indicated.

Do you think this is a low-value practice? Is this truly a "Thing We Do for No Reason?" Share what you do in your practice and join in the conversation online by retweeting it on Twitter (\#TWDFNR) and liking it on Facebook. We invite you to propose ideas for other "Things We Do for No Reason" topics by emailingTWDFNR@hospitalmedicine.org.

Disclosures: The authors have nothing to disclose.

\section{References}

1. Association APH. Deep-vein thrombosis: advancing awareness to protect patient lives. WHITE Paper. Public Health Leadership Conference on DeepVein Thrombosis.

2. Lederle FA, Zylla D, MacDonald R, Wilt TJ. Venous thromboembolism prophylaxis in hospitalized medical patients and those with stroke: a background review for an American College of Physicians Clinical Practice Guideline. Ann Intern Med. 2011;155(9):602-615. doi: 10.7326/0003-4819-155-9-20111101000008 .

3. Zubrow MT, Urie J, Jurkovitz C et al. Asymptomatic deep vein thrombosis in patients undergoing screening duplex ultrasonography. J Hosp Med. 2014;9(1):19-22. doi: 10.1002/jhm.2112.

4. Tapson VF, Decousus H, Pini M, et al. Venous thromboembolism prophylaxis in acutely ill hospitalized medical patients: findings from the International Medical Prevention Registry on Venous Thromboembolism. Chest. 2007;132(3):936-945. doi: 10.1378/chest.06-2993.

5. Guyatt GH, Eikelboom JW, Gould MK, et al. Approach to outcome measurement in the prevention of thrombosis in surgical and medical patients: Antithrombotic Therapy and Prevention of Thrombosis, 9th ed: American College of Chest Physicians Evidence-Based Clinical Practice Guidelines. Chest. 2012;141(2 Suppl):e185S-e194S. doi: 10.1378/chest.11-2289.

6. Ho KM, Tan JA. Stratified meta-analysis of intermittent pneumatic compression of the lower limbs to prevent venous thromboembolism in hospitalized patients. Circulation. 2013;128(9):1003-1020. doi: 10.1161/CIRCULATIONAHA. 113.002690

7. CLOTS (Clots in Legs Or sTockings after Stroke) Trials Collaboration, Dennis $M$, Sandercock $P$, et al. Effectiveness of intermittent pneumatic compression in reduction of risk of deep vein thrombosis in patients who have had a stroke (CLOTS 3): a multicentre randomised controlled trial. Lancet. 2013;382(9891):516-524. doi: 10.1016/S0140-6736(13)61050-8.

8. Park J, Lee JM, Lee JS, Cho YJ. Pharmacological and mechanical thromboprophylaxis in critically ill patients: a network meta-analysis of 12 trials. J Korean Med Sci. 2016;31(11):1828-1837. doi: 10.3346/jkms.2016.31.11.1828.

9. Kakkos SK, Caprini JA, Geroulakos G, et al. Combined intermittent pneumatic leg compression and pharmacological prophylaxis for prevention of venous thromboembolism. Cochrane Database Syst Rev. 2016;9:CD005258:CD005258. doi: 10.1002/14651858.CD005258.pub3.

10. Cornwell EE, 3rd, Chang D, Velmahos G, et al. Compliance with sequential compression device prophylaxis in at-risk trauma patients: a prospective analysis. Am Surg. 2002;68(5):470-473.

11. Maxwell GL, Synan I, Hayes RP, Clarke-Pearson DL. Preference and compliance in postoperative thromboembolism prophylaxis among gynecologic oncology patients. Obstet Gynecol. 2002;100(3):451-455. doi: 10.1016/ S0029-7844(02)02162-2.

12. Wood KB, Kos PB, Abnet JK, Ista C. Prevention of deep-vein thrombosis after major spinal surgery: a comparison study of external devices. J Spinal Disord. 1997; 10(3):209-214

13. Unexpected risk from a beneficial device: sequential compression devices and patient falls. PA-PSRS Patient Saf Advis. 2005 Sep;2(3):13-5.

14. Boelig MM, Streiff MB, Hobson DB, Kraus PS, Pronovost PJ, Haut ER. Are sequential compression devices commonly associated with in-hospital falls? A myth-busters review using the patient safety net database. J Patient Saf. 
2011;7(2):77-79. doi: 10.1097/PTS.0b013e3182110706

15. Ritsema DF, Watson JM, Stiteler AP, Nguyen MM. Sequential compression devices in postoperative urologic patients: an observational trial and survey study on the influence of patient and hospital factors on compliance. BMC Urol. 2013;13:20. doi: 10.1186/1471-2490-13-20.

16. Pavon JM, Williams JW, Jr, Adam SS, et al. Effectiveness of intermittent pneumatic compression devices for venous thromboembolism prophylaxis in high-risk surgical and medical patients. J Arthroplasty. 2016;31(2):524-532. doi: 10.1016/j.arth.2015.09.043.

17. Lachiewicz PF, Kelley SS, Haden LR. Two mechanical devices for prophylaxis of thromboembolism after total knee arthroplasty. A prospective, randomised study. J Bone Joint Surg Br. 2004;86(8):1137-1141. doi: 10.1302/0301-620X.86B8.15438.

18. Salzman EW, Sobel M, Lewis J, Sweeney J, Hussey S, Kurland G. Prevention of venous thromboembolism in unstable angina pectoris. N Engl J Med. 1982;306(16):991. doi: 10.1056/NEJM198204223061614.

19. Guyatt GH, Akl EA, Crowther M, Gutterman DD, Schuünemann HJ, American College of Chest Physicians Antithrombotic Therapy and Prevention of Thrombosis Panel. Executive summary: Antithrombotic Therapy and Prevention of Thrombosis, 9th ed: American College of Chest Physicians Evidence-Based Clinical Practice Guidelines. Chest. 2012;141(2 Suppl):7S-47S. doi: 10.1378/chest.1412S3.

20. Qaseem A, Chou R, Humphrey LL, Starkey M, Shekelle P, Clinical Guidelines Committee of the American College of Physicians. Venous thromboembolism prophylaxis in hospitalized patients: a clinical practice guideline from the American College of Physicians. Ann Intern Med. 2011;155(9):625-632. doi: 10.7326/0003-4819-155-9-201111010-00011.

21. Casele H, Grobman WA. Cost-effectiveness of thromboprophylaxis with intermittent pneumatic compression at cesarean delivery. Obstet Gynecol. 2006;108(3 Pt 1):535-540. doi: 10.1097/01.AOG.0000227780.76353.05.

22. Dennis M, Sandercock P, Graham C, Forbes J, CLOTS Trials Collaboration, Smith J, Smith J. The Clots in Legs or sTockings after Stroke (CLOTS) 3 trial: a randomised controlled trial to determine whether or not intermittent pneumatic compression reduces the risk of post-stroke deep vein thrombosis and to estimate its cost-effectiveness. Health Technol Assess. 2015;19(76):1-90. doi: $10.3310 /$ hta19760

23. Amazon.com. Covidien 5329 Sleeve, SCD Knee Length. https://www.amazon.com/Covidien-5329-Sleeve-Knee-Length/dp/B01BSFZM76. Accessed September 14, 2018

24. Amazon.com. 2270870 SCD Sleeve Knee Length. https://www.amazon. $\mathrm{com} / \mathrm{s} / \mathrm{ref}=\mathrm{nb} \mathrm{sb}$ noss? url=search-alias\%3Daps\&field-keywords=kendall+7325-2\&rh=i\%3Aaps\%2Ck\%3Akendall+7325-2. Accessed September 14, 2018.

25. Amazon.com. 2281540 Venodyne Advantage 610DVT. https://www.amazon.com/Individually-MODEL-610-Microtek-Medical/dp/B00IK4MUUG/ ref=sr_1_fkmr0_2?ie=UTF8\&qid=1540914574\&sr=8-2-fkmr0\&keywords=venodyne+scd. Accessed Osctober 30, 2018.

26. Amazon.com. 2339896 Venaflow System w/Battery Elite. https://www.amazon.com/indivdually-Individually-30B-B-DJO-Inc/dp/B00IK4MS3A/ref=sr_1_2?ie=UTF8\&qid=1536972486\&sr=8-2\&keywords=venaflow+elite+system. Accessed September 14, 2018.

27. Amazon.com. Covidien 29525700 Series Kendall SCD Controller. https:// www.amazon.com/Covidien-29525-700-Kendall-Controller/dp/B01BQI5BI0/ ref=sr_1_1?ie=UTF8\&qid=1536972026\&sr=8-1\&keywords=covidien +29525 . Accessed September 14, 2018.

28. Nicolaides A, Goldhaber SZ, Maxwell GL, et al. Cost benefit of intermittent pneumatic compression for venous thromboembolism prophylaxis in general surgery. Int Angiol. 2008;27(6):500-506

29. Jenkins $\mathrm{IH}$, White $\mathrm{RH}$, Amin AN, et al. Reducing the incidence of hospital-associated venous thromboembolism within a network of academic hospitals: findings from five University of California medical centers. J Hosp Med. 2016;11(Suppl 2):S22-S28. doi: 10.1002/jhm.2658.

30. Dobesh PP. Economic burden of venous thromboembolism in hospitalized patients. Pharmacotherapy. 2009;29(8):943-953. doi: 10.1592/phco.29.8.943.

31. Maynard, G. Preventing Hospital-Associated Venous Thromboembolism. A Guide for Effective Quality Improvement. AHRQ Publication No. 16-0001-EF; 2015.

32. Decousus $H$, Tapson VF, Bergmann JF, et al. Factors at admission associated with bleeding risk in medical patients: findings from the IMPROVE investigators. Chest. 2011;139(1):69-79. doi: 10.1378/chest.09-3081. 